\title{
Tuberculosis, un riesgo presente para los trabajadores en el área de la salud
}

\author{
Patricia Hidalgo Martínez ${ }^{1}$, Atilio Moreno Carrillo², \\ TATIANA Roldán Ovalle ${ }^{3}$
}

\section{Resumen}

La tuberculosis es una enfermedad de gran impacto en nuestro país y en el mundo. Aunque las estrategias de la Organización Mundial de la Salud para controlarla pueden curar casi todos los casos, la resistencia a los medicamentos y el riesgo de transmisión, especialmente en los trabajadores del área de salud, cada día cobran un mayor valor.

La tuberculosis representa un riesgo ocupacional significativo, con mayor incidencia en los trabajadores del área de salud sobre la población general, principalmente en los países en desarrollo.

El personal de la salud es fundamental en la lucha contra la tuberculosis y, por lo tanto, debe estar protegido. Los factores de riesgo de contagio incluyen la frecuencia de pacientes con tuberculosis atendidos, la función y el lugar de trabajo del trabajador del área de salud, la demora en el diagnóstico, la presencia de pacientes con cepas multirresistentes, los inadecuados sistemas de ventilación, la falta de protección adecuada contra aerosoles y el personal con compromiso de su estado inmunológico o desnutrición.

1 Médica internista, neumóloga, Hospital San Ignacio, Pontificia Universidad Javeriana, Bogotá, D.C., Colombia.

2 Médico internista; director, programa de especialización en Medicina de Urgencias, Pontificia Universidad Javeriana, Bogotá, D.C., Colombia.

3 Médica, residente de Medicina de Urgencias, Pontificia Universidad Javeriana, Bogotá, D.C., Colombia. 
Los aspectos clave para el control y la prevención de la transmisión en hospitales, son la identificación precoz de los casos, las medidas de protección ambiental y los programas efectivos para el control del personal sanitario. Las guías sobre prevención en tuberculosis recomiendan aislamiento en salas ventiladas con presión negativa y el uso de protección respiratoria para todos los trabajadores de la salud en contacto.

Palabras clave: tuberculosis, transmisión, riesgo, protección.

\section{Title}

Tuberculosis, a risk for health workers

\begin{abstract}
The impact of tuberculosis in Colombia and in the world is important. Although WHO strategies to control it can cure almost all cases, drug resistance and the risk of transmission, especially to health care workers charge a hefty price. Tuberculosis represents an important occupational risk, with higher incidence amongst health care workers compared with general population, mainly in developing countries.
\end{abstract}

The staff is crucial in the fight against tuberculosis and must be protected. Risk factors include the frequency of tuberculosis patients treated, the role and workplace of health workers, delayed diagnosis, presence of patients with multiresistant tuberculosis strains, inadequate ventilation, lack of adequate protection against aerosol and personal with altered immunological status or malnutrition.

The key points for control and prevention of tuberculosis transmission in hospitals, are earl cases identification, environmental protection measures and an effective program for control of health personnel. Tuberculosis prevention guidelines recommend patient isolation in ventilated rooms with negative pressure and use of respiratory protection for all health workers in patient contact.

Key words: tuberculosis, transmission, risk, protection.

\section{Introducción}

Se presenta el caso de un paciente con tuberculosis resistente que acudió al Servicio de Urgencias del Hospital Universitario San Ignacio y que estuvo en contacto con otros pacientes y con personal de atención en salud antes de su identificación y diagnóstico. A casos como éste se puede enfrentar cualquier institución de salud con aumento del riesgo de transmisión hospitalaria, si no se toman medidas adecuadas de protección.

Se trata de un paciente de sexo masculino, soltero, sin antecedentes relevantes previos, con sintomatología respiratoria que se inició en 2004, caracterizada por tos, fiebre no cuantificada y diaforesis nocturna. Consultó inicialmente a otra institución, donde le diagnosticaron tuberculosis pulmonar bacilífera y le iniciaron manejo antituberculoso que recibió de manera irregular durante cinco meses, persistiendo con baciloscopias positivas al finalizar el mismo.

En mayo de 2005 se le practicaron pruebas que fueron positivas para resistencia a isoniacida, rifampicina, estreptomicina y etambutol, según los reportes del Instituto Nacional de Salud, por lo que se reinició tratamiento con medicamentos cuyo nombre no recordaba el paciente. Nuevamente recibió manejo irregular e incompleto, persistiendo con baciloscopias positivas. En agosto de 
2006 se ordenó tratamiento con ciprofloxacina, etionamida, pirazinamida y rifampicina. En los controles de enero de 2007, el Instituto Nacional de Salud reportó un microorganismo multisensible. No se tienen datos de evolución, manejo o control hasta febrero de 2008, momento en el cual, por persistencia de baciloscopias positivas y nuevo reporte de resistencia, se le inició esquema con amikacina, ofloxacina, cicloserina y ácido paraaminosalicílico por tres meses, y continuó con ofloxacina, cicloserina y ácido paraaminosalicílico en una segunda fase, que recibió durante 11 meses.

Presentó persistencia y progresión de la sintomatología, dada por tos, fiebre, pérdida de peso y hemoptisis. Fue valorado en marzo de 2009 en otra institución. Por los hallazgos clínicos e imaginológicos, con radiografía y tomografía computadorizada (TC) de tórax en la que se observaba una cavidad en el segmento posterior del lóbulo superior izquierdo y árbol en gemación del mismo lado, lo remitieron al Hospital Universitario San Ignacio para practicar lobectomía y reiniciar el tratamiento.

Ingresó a la institución con hallazgos en el examen físico de adenopatías móviles no dolorosas en la región submandibular izquierda, ruidos respiratorios disminuidos en ambas bases y roncus bilaterales.

Con diagnóstico de tuberculosis pulmonar multirresistente y persistencia de BAAR positivos, por cultivo para identificación y antibiograma, se inició el tratamiento ajustado por peso con las siguientes dosis diarias: $15 \mathrm{mg} / \mathrm{kg}$ intramusculares de amikacina, $15 \mathrm{mg} / \mathrm{kg}$ de etionamida, $400 \mathrm{mg}$ de moxifloxacina, 10 a $15 \mathrm{mg} / \mathrm{kg}$ de cicloserina, $15 \mathrm{mg} / \mathrm{kg}$ de etambutol y $20 \mathrm{mg} / \mathrm{kg}$ de pirazinamida. Fue sometido a escisión del segmento apical de lóbulo inferior izquierdo, cuyo estudio histopatológico reportó neumonía granulomatosa de probable origen tuberculoso. Su evolución posoperatoria fue adecuada. Fue dado de alta y se observó evolución hacia la mejoría en su control ambulatorio de agosto de 2009.
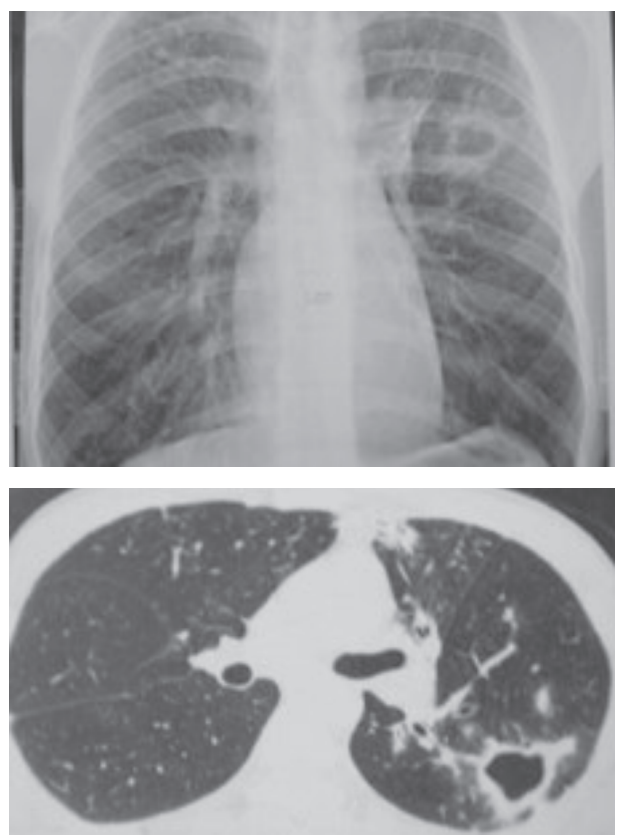

Figura 1. Se observa cavidad de paredes ligeramente engrosadas en el lóbulo inferior izquierdo con pérdida de volumen de los lóbulos superiores. 


\section{Discusión}

A pesar de las políticas desarrolladas para su control y para evitar su incremento en la comunidad, la tuberculosis continúa siendo en la actualidad un grave problema de salud pública en Colombia y en el mundo[1]. La Organización Mundial de la Salud (OMS) estima que cada año se producen entre 8 y 10 millones de casos nuevos y 3 millones de muertes por esta causa. Los datos actuales indican que más de la tercera parte de la población mundial se encuentra infectada por Mycobacterium tuberculosis.

En 2007, 202 de 212 países y territorios del mundo comunicaron a la OMS datos de notificación de tuberculosis, correspondientes a 2006. Para ese año, se notificaron 5,1 millones de casos nuevos, de una cifra estimada de 9,2 millones. El número estimado de nuevos casos de tuberculosis en 2008 fue de 9 millones, aproximadamente, con una prevalencia de casi 13,5 millones de casos y, en promedio, 1,4 millones de muertes de personas negativas para VIH $\mathrm{y}$, además, 0,5 millones de muertes por tuberculosis de pacientes positivos para VIH. El número de casos notificados de tuberculosis en 2008 fue de casi 6 millones, equivalentes a 55 a $67 \%$ de todos los casos de incidencia[2]. En Bogotá, en 2008 se notificaron 1.131 casos de tuberculosis y 1.147 para 2009 , con predominio del tipo con compromiso pulmonar.
La OMS lanzó en 2006 la estrategia Alto a la tuberculosis, diseñada para alcanzar las metas de impacto de 2015, así como las metas en materia de detección de casos y éxito terapéutico. Este plan mundial detalla la escala en la que deben aplicarse los seis componentes de la estrategia Alto a la tuberculosis para alcanzar esas metas, así como los fondos necesarios, para cada año entre 2006 y 2015[3].

La tuberculosis es una enfermedad bacteriana infectocontagiosa, producida por micobacterias del complejo Mycobacterium (M. tuberculosis, M. bovis y $M$. africanum).

En Berlín, en 1882, Roberto Koch aisló el bacilo tuberculoso y demostró su carácter patógeno.

M. tuberculosis es el principal agente causal de tuberculosis humana. Es un bacilo aerobio, inmóvil, que protegido de la luz solar puede permanecer viable en el esputo durante semanas o meses. La transmisión se efectúa por vía aérea, al inhalar partículas de esputo que exhala el enfermo al toser (la forma más efectiva de transmisión), hablar o estornudar[4] pero requiere combinación con otros factores, como concentración suficiente de bacilos suspendidos en el aire, huésped sensible y tiempo suficiente del huésped respirando aire contaminado.

El riesgo es universal, para todas las edades, sexos y razas; sin embargo, la 
desnutrición, el hacinamiento, las malas condiciones higiénicas, la infección por $\mathrm{VIH}$, los medicamentos inmunosupresores, las alteraciones hormonales, la silicosis, el alcoholismo, la drogadicción y la indigencia, entre otros, aumentan la sensibilidad de las personas a la enfermedad.

El riesgo de tuberculosis hospitalaria, ya sea por transmisión a otros pacientes o hacia el propio personal de salud, ha sido un hecho bien conocido, y la aparición de casos de cepas resistentes ha desviado la atención sobre este tema a nivel mundial[5].

El grupo de trabajadores de la salud es vulnerable a adquirir la enfermedad y su riesgo ocupacional está determinado directamente por la exposición a pacientes infectados. Esta situación es especialmente alarmante por el reporte de brotes de tuberculosis adquirida en los hospitales en países en desarrollo, tanto entre trabajadores de la salud como en pacientes[6].

Antes del advenimiento de los fármacos antituberculosos, el riesgo de los trabajadores de la salud de adquirir tuberculosis era extremadamente elevado, pero declinó rápidamente después de la década de los cincuenta, al disminuir las tasas de incidencia en la población general. Infortunadamente, esto ha llevado a descuidos en la aplicación de los principios de control de la infección tuberculosa hospitalaria y, en ocasiones, al abandono de estas medidas[7,8].
Los reportes provenientes de los países con elevadas tasas de tuberculosis han demostrado que los trabajadores de la salud a cargo de la atención de pacientes con tuberculosis presentan, actualmente, un elevado riesgo de adquirir la infección por $M$. tuberculosis y de desarrollar la enfermedad. Los trabajadores de la salud en los servicios de urgencias tienen hasta 20 veces más riesgo de desarrollar tuberculosis activa que el personal administrativo de la misma unidad, debido principalmente a que los pacientes con tuberculosis que allí acuden suelen presentar enfermedad avanzada asociada a baciloscopia positiva y típicamente no son diagnosticados ni aislados sino hasta varias horas o días después de su ingreso, o porque ocultan su diagnóstico por temor a ser rechazados, lo que incrementa el riesgo de contagio del personal laboral[9].

Antes del tratamiento de la tuberculosis, el riesgo estimado de infección anual alcanzaba el $80 \%$ del personal. En la era posterior al tratamiento antituberculoso y durante la existencia de brotes, este riesgo oscila entre 14 y $55 \%$ para infección latente y entre 2,2 y $8,4 \%$ para tuberculosis clínica.

Entre los factores que explican las diferencias en el riesgo de infección o desarrollo de tuberculosis activa, se destacan el volumen de pacientes con tuberculosis atendidos, la función u ocupación del personal de salud, el lugar de trabajo del trabajador del área de 
salud, la demora en el diagnóstico de los pacientes con tuberculosis, las deficiencias en la ventilación ambiental, la aplicación o falta de medidas de aislamiento para aerosoles, las barreras de protección que usa el personal clínico y la existencia de trabajadores del área de salud con alguna condición de inmunosupresión o desnutrición[10].

Se ha podido establecer que no todos los casos de tuberculosis activa en el personal de salud son adquiridos en el medio hospitalario. Hay una fracción de ellos que ocurre por reactivación de una infección latente antigua. En un estudio reciente De Vries et al. se logró determinar que sólo $42 \%$ de los casos observados durante cinco años en trabajadores del área de salud de los Países Bajos fue por adquisición hospitalaria, $28 \%$ por contagio en la comunidad y el $30 \%$ restante por adquisición remota[11]. En México, durante 1992-1998, se vigilaron 1.617 trabajadores de la salud y se encontró que la prueba de la tuberculina al ingreso fue positiva en $39,6 \%$ de los casos, con una tasa de conversión a los 12 meses de $20 \%[12]$.

La incidencia de tuberculosis en trabajadores del área de salud de los hospitales argentinos es 3,7 veces mayor que la de la población general. En el periodo comprendido entre 2001 y 2004 , se diagnosticaron 68 casos de tuberculosis en trabajadores del área de salud (incidencia anual media, 111,3/100.000) y la tuberculosis ocupacional fue más frecuente en personal de enfermería $(51,5 \%)$ [13]. En nuestra institución, el $34,8 \%$ de los trabajadores del área de salud evaluados tienen tuberculosis latente[14].

Diferentes condiciones del paciente aumentan el riesgo de contagio del personal de salud. Entre éstos se encuentran la presencia de tos, cavidades pulmonares, compromiso laríngeo, administración incorrecta o abandono del tratamiento y procedimientos que estimulen la producción de aerosoles, como fibrobroncoscopia, expectoración inducida y nebulizaciones[15].

La presentación clínica de tuberculosis activa en el personal de salud no difiere de la de otros pacientes, con predominio de la forma pulmonar con tos, expectoración, hemoptisis, fiebre y pérdida de peso[16]; sin embargo, algunas publicaciones describen el predominio de las formas extrapulmonares, principalmente la ganglionar[17]. El trabajador del área de salud debe conocer los síntomas de la tuberculosis y someterse a un examen médico que incluya pruebas para detectarla, por lo menos, una vez al año.

Existen estrategias que permiten hacer el diagnóstico en ausencia de síntomas y la búsqueda activa de trabajadores del área de salud con tuberculosis latente y tuberculosis activa. Entre ellas se encuentran la prueba de 
conversión de tuberculina y la prueba de liberación de interferón gamma[18]. La primera consiste en una inyección intramuscular de derivado proteico purificado (Purified Protein Derivative, PPD), para identificar a las personas que se han sensibilizado a los antígenos micobacterianos por estar infectadas con M. tuberculosis u otras micobacterias no tuberculosas, o por haber recibido la vacuna BCG. Pero la tamización con esta prueba para el trabajador del área de salud en países en desarrollo es complicada, porque produce resultados difíciles de interpretar debido al amplio uso de la vacuna BCG y la reacción cruzada con micobacterias atípicas, y porque requiere de muchos recursos para su práctica e interpretación[19].

La segunda prueba está basada en la liberación de interferón gamma por parte de los linfocitos $\mathrm{T}$ de individuos previamente sensibilizados con antígenos tuberculosos, al reencontrarse con antígenos propios de M. tuberculosis. Tiene mayor especificidad que la PPD debido a que su resultado no se afecta por la vacuna BCG. El principal inconveniente es su alto costo[20].

Las medidas de prevención recomendadas para evitar la infección en el trabajador del área de salud se dividen en tres componentes principales[21]. El primero son las medidas administrativas, que deben incluir: identificación de áreas de riesgo, sospecha y diagnóstico precoz de pacientes, aislamiento de pacientes con alta sospecha o diagnóstico de tuberculosis, desinfección de equipos de riesgo (fibrobroncoscopio), educación y entrenamiento al personal de salud, tamización para el diagnóstico de infecciones y enfermedad en los trabajadores del área de salud. El control de la transmisión es eficaz si todas las personas que trabajan en una institución comprenden la importancia de las políticas de control de las infecciones y su función en la ejecución de las mismas. Cada trabajador de salud debe recibir instrucción apropiada para su categoría de trabajo y educación de forma continua[22].

El segundo componente está relacionado con la infraestructura, mediante la implementación de sistemas de ventilación o depuración de aire en los lugares de permanencia de pacientes bacilíferos pulmonares. Una variedad de medidas pueden usarse para reducir el número de aerosoles infecciosos en el ambiente de trabajo, como maximizar la ventilación natural mediante ventanas abiertas, o métodos más complejos que incluyen el uso de ventilación mecánica en lugares de aislamiento para producir presión negativa y extracción de partículas infecciosas[23].

El tercero incluye la protección respiratoria personal. Hay diferencias importantes entre un tapabocas y una mascarilla. El tapabocas (de tela o papel) evita la propagación de los microorganismos de la persona que lo lleva 
puesto hacia otros, pero no protege contra la inhalación de partículas infecciosas a la persona sana que lo porta. Aunque no es una intervención de alta prioridad, los tapabocas pueden usarse para reducir los aerosoles generados por los pacientes tuberculosos. Los tapabocas generalmente tienen capacidad de filtración limitada y su mala colocación permite la entrada libre de $M$. tuberculosis[24,25]. Dado que esta protección es costosa, su uso puede ser apropiado en áreas de alto riesgo de la institución.

El uso de mascarillas con eficiencia del filtro de, al menos, 95\% (N95) para partículas muy pequeñas, permite al trabajador de la salud tener una menor exposición a los aerosoles cuando atiende pacientes con tuberculosis pulmonar bacilífera. Permite la retención y filtración de partículas menores de $5 \mathrm{ìm}$, pero deben ser utilizadas apropiadamente sin filtraciones de aire por los costados y con previo entrenamiento para su uso. Son desechables, pero pueden reutilizarse si se guardan adecuadamente.

Otras acciones que se deben tener en cuenta para la protección del trabajador del área de salud incluyen: adecuado entrenamiento, identificación de pacientes con tos $\mathrm{y}$, principalmente, evitar que trabajadores del área de salud con infección por VIH o desnutrición se involucren en el proceso de atención de pacientes con tuberculosis activa.

Sin medidas administrativas y ambientales de control, los implementos de protección personal no actuarán adecuadamente contra las transmisiones. La prueba de la eficacia de los componentes individuales es que el riesgo de transmisión disminuye de forma importante si se combinan todas las medidas[26].

Como parte de la prevención primaria de la transmisión al personal de salud, se debe someter a un examen precoz a todos los pacientes que lleguen con tos crónica (tos de tres o más semanas de duración), fiebre, pérdida de peso y hemoptisis, o que hayan tenido contacto con una persona con tuberculosis. El personal de salud debe explicarles a los pacientes que la seguridad es la meta y que la tamización forma parte de la atención de buena calidad. Los pacientes en los que se sospecha tuberculosis deben ser atendidos de forma prioritaria para hacer el diagnóstico e iniciar un tratamiento precoz.

En los establecimientos de salud con salas de hospitalización, los pacientes sintomáticos respiratorios deben agruparse en una sala o lugar adecuadamente ventilado e independiente de las salas generales. Estos pacientes no deben permanecer en pasillos estrechos y mal ventilados.

Al personal de salud se le debe proveer dispositivos apropiados de protección respiratoria y a los trabajadores positivo para VIH se les debe brindar la oportunidad de reducir al 
mínimo su exposición a pacientes con tuberculosis, ofreciéndoles funciones diferentes.

Debe incorporarse la enseñanza al trabajador del área de salud de los métodos para evitar la infección por tuberculosis y brindar capacitación general para el control de las infecciones en los hospitales y establecimientos sanitarios.

\section{Bibliografía}

1. Galvis V, Bustamante M, Sarmiento C. Guías de atención de la tuberculosis pulmonar y extrapulmonar. Bogotá: Ministerio de Salud. Fecha de consulta: octubre de 2009. Disponible en: http:// www.col.opsoms.org/prevencion/ tuberculosis/guia_tuberculosis.pdf.

2. World Health Organization. Global tuberculosis control: a short update to the 2009 report. Geneve: World Health Organization. 2009;1.

3. Organización Mundial de la Salud. Tuberculosis [Sede web]. Centro de prensa. [Revisada marzo de 2007]. Fecha de consulta: octubre de 2009. Disponible en: http://www.who.int/ mediacentre/factsheets/fs 104/es/ index.html

4. Palmero D, Laniado R, Jave O, Barrera L, Caminero J. Guías latinoamericanas de diagnóstico y tratamiento de la tuberculosis farmacorresistente [Sede Web] Asociación Latinoamericana de Tórax (ALAT). Fecha de consulta: octubre de 2009. Disponible en: http:// www.intramed.net/UserFiles/pdf/ 57367.pdf
5. Fica A, Cifuentes M, Ajenjo C, Jemenao I, Zambrano A, Febré N. Tuberculosis en el personal de salud. Revista Chilena de Infectología. 2008;25:243-55.

6. Ostrsoky-Zeichner L, Rangel-Frausto MS, García E, Vázquez A, Ibarra J, Ponce de León S. Tuberculosis en trabajadores de la salud: importancia de los programas de vigilancia y control. Salud Pública de México. 2000;42: 48-52.

7. Cronin WA. Frontline health careworkers continue to be all high risk for tuberculosis. Int J Tuberc Lung Dis. 2005;9(11):1180.

8. Joshi R, Reingold AL, Menzies D, Pai M. Tuberculosis among health-care workers in low- and middle-income countries: a systematic review. Plos Med. 2006;3:2376-91.

9. Dimitrova B, Hutchings A, Atun R, Drobniewiski F, Marchenko G, Zakharova S. Increased risk of tuberculosis among health care workers in Samara Oblast, Russia: Analysis of notification data. Int J Tuberc Lung Dis. 2005;9:43-8.

10. Laniado-Laborin R. Cabrales-Vargas N. Tuberculosis in healthcare workers at a general hospital in Mexico. Infect Control Hosp Epidemiol. 2006;27: 449-52.

11. De Vries G, Sebeck MM, Lambregts-van Weezenbeek CS. Healthcare workers with tuberculosis infected during work. Eur Respi J. 2006;28:1216-21.

12. Ostrosky-Zeichner L. Tuberculosis en trabajadores de la salud: importancia de los programas de vigilancia y control. Salud Pública de México. 2000;42:48-52. 
13. Trabajadores de la Salud, Grupo de Trabajo Argentino en Tuberculosis. Análisis de la incidencia de tuberculosis entre los trabajadores de la salud de hospitales argentinos. Buenos Aires. 2006.

14. Ruiz A, Hidalgo P, Álvarez C, Fernández $\mathrm{D}$, Manrique L. Resultados de PPD en trabajadores del Hospital Universitario San Ignacio. XX Congreso Colombiano de Medicina Interna, Bogotá, marzo de 2008.

15. Centers for Disease Control and Prevention. Guidelines for preventing the transmission of Mycobacterium tuberculosis in healthcare facilities. MMWR Morb Mortal Wkly Rep. 2005; 54:1-141.

16. Díaz-Castrillo AO, Dueñas-Mojena D, Lazo-Alvarez MA, Borroto-Gutiérrez S, González-Ochoa E. Tuberculosis en trabajadores de la salud del Hospital Psiquiátrico de la Habana, 1997-2003. Rev Panam Infectol. 2005; 7:22-6.

17. Alonso-Echanove J, Granich R M, Laszlo A, Chu G, Borja N, Blas R, et al. Occupational transmission of $\mathrm{Myco}$ bacterium tuberculosis to health care workers in a university hospital in Lima, Perú. Clin Infect Dis. 2001;33:589-96.

18. Fica A, Cifuentes M, Ajenjo C, et al. Tuberculosis en el personal de salud. Revista Chilena de Infectología. 2008; 25:243-55. Esta bibliografía también está repetida. Es la misma 5.

19. Granich R, Binskin N, Jarvis W, Simone P. Normas para la prevención de la transmisión de la tuberculosis en los establecimientos de asistencia sanitaria en condiciones de recursos limitados. Ginebra, Suiza: OMS, Programa de enfermedades transmisibles. 2002.
20. Richeldi L. An update on the diagnosis of tuberculosis infection. Am J Respir Crit Care Med. 2006;174:736-42.

21. Centers for Disease Control and Prevention. Guidelines for preventing the transmission of Mycobacterium tuberculosis in healthcare facilities. MMWR Morb Mortal Wkly Rep. 2005; 54(RR17):1-141.

22. Díaz-Castrillo AO, Dueñas-Mojena D, Lazo-Álvarez MA, Borroto-Gutiérrez S, González-Ochoa E. Tuberculosis en trabajadores de salud del Hospital Psiquiátrico de La Habana, 1997-2003. Rev Panam Infectol. 2005;7:22-6.

23. Jensen PA, Lambert LA, Lademarco MF, Ridzon R. Guidelines for preventing the transmission of Mycobacterium tuberculosis in health care setting. Morb Mort Week Rep. 2005;4:1-141.

24. Granich R, Binskin N, Jarvis W, Simone P. Normas para la prevención de la transmisión de la tuberculosis en los establecimientos de asistencia sanitaria en condiciones de recursos limitados. Programa de enfermedades transmisibles OMS. Ginebra, Suiza. 2002.

25. Centers for Disease Control and Prevention. Guidelines for preventing the transmission or mycobacterium tuberculosis in health-care facilities, 1994. Morb Mortal Wkly Rep. 1994;43: $1-132$.

26. Humphreys H. Control and prevention of healthcare-associated tuberculosis: The role of respiratory isolation and personal respiratory protection. Journal of Hospital Infection. 2007;66:1-5. 